\title{
Range resolution improvement of Eyesafe Ladar Testbed (ELT) measurements using sparse signal deconvolution
}

\author{
Scott E. Budge, Jacob H. Gunther \\ Dept. of Electrical and Computer Engineering, Utah State University, \\ Logan, UT 84322-4120, (435) 797-3433
}

\begin{abstract}
The Eyesafe Ladar Test-bed (ELT) is a experimental ladar system with the capability of digitizing return laser pulse waveforms at $2 \mathrm{GHz}$. These waveforms can then be exploited off-line in the laboratory to develop signal processing techniques for noise reduction, range resolution improvement, and range discrimination between two surfaces of similar range interrogated by a single laser pulse.

This paper presents the results of experiments with new deconvolution algorithms with the hoped for gains of improving the range discrimination of the ladar system. The sparsity of ladar returns is exploited to solve the deconvolution problem in two steps. The first step is to estimate a point target response using a database of measured calibration data. This basic target response is used to construct a dictionary of target responses with different delays/ranges. Using this dictionary ladar returns from a wide variety of surface configurations can be synthesized by taking linear combinations. A sparse linear combination matches the physical reality that ladar returns consist of the overlapping of only a few pulses. The dictionary construction process is a pre-processing step that is performed only once.

The deconvolution step is performed by minimizing the error between the measured ladar return and the dictionary model while constraining the coefficient vector to be sparse. Other constraints such as the nonnegativity of the coefficients are also applied. The results of the proposed technique are presented in the paper and are shown to compare favorably with previously investigated deconvolution techniques.
\end{abstract}

Keywords: lidar, ladar, waveform processing, sparse deconvolution, surface response, range resolution enhancement

\section{INTRODUCTION}

The availability of waveform digitizing ladar systems is stimulating the growth of signal processing techniques to improve the resolution and reduce the range error of ladar measurements. Other performance parameters, such as false alarm rate and signal detection thresholds can be optimized since the returned waveform can be observed.

There are several techniques that have been applied to ladar waveforms to improve performance. Gaussian decomposition is a commonly used method that provides an estimate of amplitude and timing of return pulses by fitting the waveform with a sum of Gaussian pulses. ${ }^{1-5}$

Other methods have been developed that approach the problem as an ideal "surface response" that have been blurred by convolution with the laser pulse waveform. ${ }^{5-7}$ The goal of deconvolution is to remove the effects of convolution and obtain the original surface response.

In a previous work, Neilsen et al investigated several different methods for deconvolution to find the surface response when two surfaces are offset by a known amount. ${ }^{8}$ The minimum distance that was resolvable using their method and ladar system was a separation of $11 \mathrm{~cm}$. The best results were obtained using a non-negative leastsquares (LS) technique based on the Levenberg-Marquardt algorithm. ${ }^{9}$ The resolution of the system without waveform processing was $29 \mathrm{~cm}$.

There has been a recent interest in applying the theory of compressive sensing and reconstruction to improve the methods for deconvolution. ${ }^{10}$ These methods attempt to deconvolve the signal by computing the LS solution with the additional constraint that the solution be as sparse as possible. This allows an additional parameter for regularization of the solution. 
In general, our research is focused on improving ladar range resolution to discriminate surfaces that are closely spaced in range (the sub-15 centimeter regime). This paper presents results of exploring a family of deconvolution methods that exploit the sparsity of the ladar returns. Using these methods, the deconvolution problem can be posed as a LS optimization problem with a special form of regularization involving a free parameter. We explore the solution space over the useful range of this free parameter. Interestingly, this technique subsumes non-negative LS as a special case. Therefore, the work reported here may be viewed as a generalization of our previous results.

The paper is organized as follows. Section 2 provides an introduction to the system used to collect data. Section 3 outlines the techniques used for signal processing. Section 4 contains the experiment used to compare methods, and conclusions are given in Section 5.

\section{THE EYESAFE LADAR TEST-BED}

The Vehicle Integrated Sensor Suite for Targeting Applications (VISSTA) van was developed to provide a mobile sensor platform for multiple modes of data collection. ${ }^{11}$ As shown in Fig. 1, the ELT, mounted in the VISSTA van, has a single sensor flying spot laser scanner that sits inside a movable turret on top of the van. A color camera is mounted coaxially with the eyesafe laser to allow the operator to observe the field of view of the ELT scanner. This also enables $3 \mathrm{D}$ texel images to be formed by fusing the camera image with the 3D point cloud. ${ }^{12}$

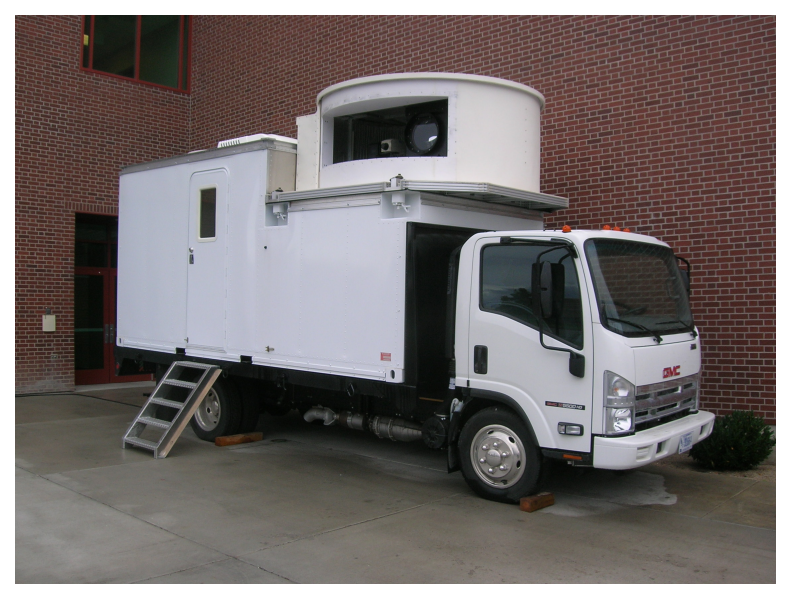

Figure 1. The VISSTA van. The ELT aperture can be seen at the right side in the turret behind the cab.

The ELT is capable of scanning a $4^{\circ} \times 4^{\circ}$ field of view at up to a $100 \mathrm{kHz}$ pulse rate. The range gate of the digitizer can be set up to $500 \mathrm{~m}$ for a $20 \mathrm{kHz}$ pulse rate. The ELT digitizes the return pulse waveform at $2 \mathrm{GHz}$ to enable offline data analysis. The laser pulse width is $1.5 \mathrm{~ns}$ at full-with half-maximum (FWHM), resulting in waveforms sampled at two times the Nyquist frequency.

\section{SIGNAL PROCESSING}

The signal model for the ELT ladar receiver is given in Fig. 2.8 The transmitted pulse is detected using electronics that are separate from the receiver system, which is of the same design. Since these are separate systems, different transfer functions $(H(s))$ are used in modeling the digitized exiting pulse and the return pulse.

The input to the system model is a Dirac delta function $\delta(t)$, which is the ideal transmitted pulse shape. The actual transmitted laser pulse is created by convolving the delta function with the laser pulse transfer function $P(s)$. The pulse then travels to the target, where it is convolved with the target surface response $S(s)$. Noise sources from backscattered radiation, solar background, dark current, and receiver electronics are added to the signal as white noise $n_{t}(t)$. Amplitude dependent (multiplicative) shot noise from the avalanche photodiode (APD) is also added. The signal then proceeds through the receiver electronics $H(s)$. Finally, quantization and measurement noise $n_{q}(n)$ in the A/D converter is added to obtain the stored waveform $r(n)$. 


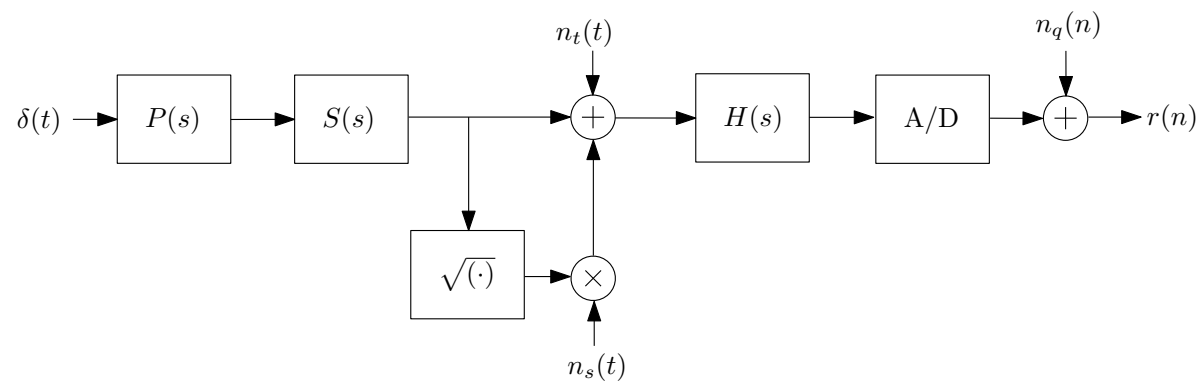

Figure 2. Signal model for the received waveform.

The model was simplified by assuming the multiplicative noise can be modelled as additive white noise with variance proportional to the average value of the pulse waveform. This leads to the frequency-domain sampled signal

$$
R(f)=P(f) S(f) H(f)+N(f) H(f)+N_{q}(f),
$$

where

$$
\begin{gathered}
N(f)=N_{t}(f)+\sqrt{\overline{p_{s}(t)}} N_{s}(f), \\
p_{s}(t)=p(t) * s(t),
\end{gathered}
$$

and where $\overline{p_{s}(t)}$ is the time average of $p_{s}(t)$ during the duration of the pulse, $f$ is the normalized discrete frequency, $N_{t}(f)$ and $N_{s}(f)$ are white noise, and $p(t)$ and $s(t)$ are the inverse Laplace transforms of $P(s)$ and $S(s)$ respectively.

The signal processing goal is to recover the discrete-time surface response $s(n)$ from the received waveform $r(n)$. This is achieved by the process of filtering, interpolation, deconvolution, and range discrimination as shown in Fig. 3, where $z$ is used to denote a discrete-time transfer function. The interpolation upsampling rate $N$ used in this work was 10. See Neilsen et al for details. ${ }^{8}$ Since this paper presents a study of sparse deconvolution methods, only the deconvolution methods will be described here.

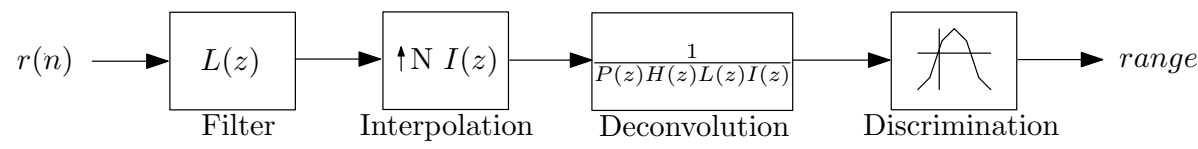

Figure 3. Processing method used in the VISSTA ELT.

\subsection{Deconvolution}

Deconvolution allows us to determine the surface response of the target and use it to potentially obtain an estimate of the range to multiple surfaces within the beam footprint. Applying the lowpass filters $L(z)$ and $I(z)$ to (1) leads to

$$
R(z) L(z) I(z)=\left(P(z) S(z) H(z)+N(z) H(z)+N_{q}(z)\right) L(z) I(z),
$$

which can be rearranged to obtain the target surface response

$$
S(z)=\frac{\tilde{R}(z)}{\tilde{H}(z)}+\tilde{N}(z)
$$

where

$$
\begin{gathered}
\tilde{R}(z)=R(z) L(z) I(z), \\
\tilde{H}(z)=P(z) H(z) L(z) I(z),
\end{gathered}
$$


and

$$
\tilde{N}(z)=\frac{N(z)}{P(z)}+\frac{N_{q}(z)}{P(z) H(z)}
$$

Deconvolution is possible only if $\tilde{R}(z)$ and $\tilde{H}(z)$ are known and effects from $\tilde{N}(z)$ can be minimized. $\tilde{R}(z)$ is the measured waveform after filtering and interpolation. To obtain $\tilde{R}(z)$, the locations of the returns in the signal are found using a simple threshold, and $r(n)$ is segmented into signal segments around the detected return. Each signal segment can then be processed individually.

$\tilde{H}(z)$ (the blur function in the dictionary of ladar returns) is obtained by holding the scanner stationary while aimed at a flat surface oriented normal to the ELT, and averaging many returns together. Averaging substantially reduces noise and provides an accurate estimate of the blur function. Alternatively, the transmitted pulse for each shot could be used to estimate the blur function, ${ }^{5}$ but noise on each transmitted pulse waveform, combined with differences in the electronics in the ELT used in the capture of the transmitted pulse, produce a poorer estimate of the blur than the averaging method used.

\subsubsection{Family of Least Squares Deconvolution Methods}

Least squares deconvolution methods are based upon a model in which the measured waveform $r(t)$ consists of a superposition of delayed and scaled replicas of a basic pulse $b(t)$ which represents the response of the ladar system to an idealized point target reflector at zero range. The model may be expressed mathematically as

$$
r(t)=\int s(\tau) b(t-\tau) d \tau+e(t)
$$

where $e(t)$ represents noise plus unmodeled effects and $s(\tau)$ is the scene spatial reflectivity structure. Note that $s(\tau) \geq 0$ because negative reflectivity is physically meaningless.

Variations on this basic model are possible. If the laser has perpendicular incidence on a flat surface $s(\tau)=$ $a_{1} \delta\left(\tau-\tau_{1}\right)$, then the signal model (9) simplifies to

$$
r(t)=a_{1} b\left(t-\tau_{1}\right)+e(t), \quad a_{1} \geq 0,
$$

where the pulse amplitude $a_{1}$ accounts for range loss and other losses and $\tau=2 R / c$ is the two-way time delay to the target surface. In the single-surface case, the deconvolution problem is solved by estimating $a_{1}$ and $\tau_{1}$ given measurements of $r(t)$.

Another special case, which is especially relevant to the current work, is the two-surface model $s(t)=$ $a_{1} \delta\left(\tau-\tau_{1}\right)+a_{2} \delta\left(\tau-\tau_{2}\right)$ which leads to

$$
r(t)=a_{1} b\left(t-\tau_{1}\right)+a_{2} b\left(t-\tau_{2}\right)+e(t), \quad a_{1}, a_{2} \geq 0 .
$$

This model holds when the footprint of the laser falls across an edge. Reflections from both near far surfaces are measured with time delays related to the range to the two surfaces. The sizes of the amplitudes $a_{1}$ and $a_{2}$ are functions of the fraction of laser footprint incident on each surface as well as the surface reflectivities. In the two-surface case, the deconvolution problem is solved by estimating $a_{1}, a_{2}, \tau_{1}$ and $\tau_{2}$ given measurements of $r(t)$.

The full generality of the integral model is needed to account for oblique incidence angles. In this case, a continuum of pulses are reflected back and are measured by the ladar system. In the following the one and two surface models are investigated in greater depth.

As described previously, the ELT samples at $2 \mathrm{GHz}$ leading to $7.5 \mathrm{~cm}$ of range resolution followed by upsampling by a factor of 10 leading to $7.5 \mathrm{~mm}$ resolution. After sampling and interpolation by 10, the single-surface model (10) becomes

$$
r(n T)=a_{1} b\left(n T-\tau_{1}\right)+e(n T), \quad a_{1} \geq 0,
$$

where $T$ is the sample period. 
Due to oversampling and assuming that the $b(t)$ is a smooth function, the point target response delayed by a non-integer multiple of the sample time $\tau_{1}$ may be approximated as the superposition of the two nearest integer delays

$$
b\left(n T-\tau_{1}\right)=b\left(\left[n-\frac{\tau_{1}}{T}\right] T\right) \approx \alpha_{1} b\left(\left[n-k_{1}\right] T\right)+\beta_{1} b\left(\left[n-\left(k_{1}+1\right)\right] T\right) .
$$

Substituting this approximation into the one and two surface models leads to

$$
\begin{aligned}
& r(n T)=\alpha_{1} b\left(\left[n-k_{1}\right] T\right)+\beta_{1} b\left(\left[n-\left(k_{1}+1\right)\right] T\right)+e(n T), \\
& r(n T)=\alpha_{1} b\left(\left[n-k_{1}\right] T\right)+\beta_{1} b\left(\left[n-\left(k_{1}+1\right)\right] T\right)+\alpha_{2} b\left(\left[n-k_{2}\right] T\right)+\beta_{2} b\left(\left[n-\left(k_{2}+1\right)\right] T\right)+e(n T),
\end{aligned}
$$

subject to $\alpha_{i}, \beta_{i} \geq 0$. The deconvolution problem is to estimate the parameters $\alpha_{i}, \beta_{i}$ and $k_{i}$. In practice, the number of surfaces is not known and must be determined.

Introducing integer delays into the model enables a convenient matrix-vector formulation. This is accomplished as follows. Stack samples of the measured waveform into the vector $\mathbf{r}$, and arrange samples of the delayed point response into the vector $\mathbf{b}_{k}$,

$$
\mathbf{r}=\left[\begin{array}{c}
r(0 T) \\
r(1 T) \\
r(2 T) \\
\vdots \\
r([N-1] T)
\end{array}\right], \quad \mathbf{b}_{k}=\left[\begin{array}{c}
b([0-k] T) \\
b([1-k] T) \\
b([2-k] T) \\
\vdots \\
b([N-1-k] T)
\end{array}\right]
$$

Define an error vector e similar to $\mathbf{r}$. Then the models (14) and (15) can be written as

$$
\begin{array}{lrl}
\mathbf{r}=\alpha_{1} \mathbf{b}_{k_{1}}+\beta_{1} \mathbf{b}_{k_{1}+1}+\mathbf{e}, & \alpha_{1}, \beta_{1} \geq 0 \\
\mathbf{r}=\alpha_{1} \mathbf{b}_{k_{1}}+\beta_{1} \mathbf{b}_{k_{1}+1}+\alpha_{2} \mathbf{b}_{k_{2}}+\beta_{2} \mathbf{b}_{k_{2}+1}+\mathbf{e}, & \alpha_{1}, \alpha_{2}, \beta_{1}, \beta_{2} \geq 0 .
\end{array}
$$

Collect the delayed response vectors into the columns of a matrix

$$
\mathbf{B}=\left[\begin{array}{lllll}
\mathbf{b}_{0} & \mathbf{b}_{1} & \mathbf{b}_{2} & \cdots & \mathbf{b}_{M-1}
\end{array}\right] .
$$

Note that the $\mathbf{B}$ matrix will be wide, i.e. have more columns than rows. Using these definitions, the one and two surface models in (14) and (15) may be written as

$$
\mathbf{r}=\mathbf{B s}+\mathbf{e}, \quad \mathbf{s} \geq 0,
$$

where the $\mathbf{s}$ vector is mostly zeros with a few non-zero coefficients placed in a fashion to select and scale the appropriate columns of the $\mathbf{B}$ matrix,

$$
\mathbf{s}=\left[\begin{array}{lllllllllllll}
0 & \cdots & 0 & \alpha_{1} & \beta_{1} & 0 & \cdots & 0 & \alpha_{2} & \beta_{2} & 0 & \cdots & 0
\end{array}\right]^{T},
$$

where the $\alpha_{1}, \beta_{1}$ values occupy the $k_{1}, k_{1}+1$ positions and the $\alpha_{2}, \beta_{2}$ values occupy the $k_{2}, k_{2}+1$ positions in the vector. Vectors such as $\mathbf{s}$ in (21) having only a few non-zero elements are called sparse vectors.

The matrix-vector model (20) subsumes the single-surface and double-surface models as special cases. It is also more general in that more than two surfaces can be modeled as well. As the number of surfaces increases, the vector becomes less and less sparse. Because the laser footprint is small spatially, the probability of large numbers of surfaces is very small. The most likely scenarios are one and two surfaces. Therefore, a complete specification of the model for the received data must include a statement about the sparsity of $\mathbf{s}$, such as

$$
\mathbf{r}=\mathbf{B} \mathbf{s}+\mathbf{e} \quad \text { subject to } \mathbf{s} \geq 0 \text { and } \mathbf{s} \text { is sparse. }
$$

Notice that the statement about the sparsity of $\mathbf{s}$ places no constraints on the location of the non-zeros in $\mathbf{s}$ or the exact number of non-zeros. 
The deconvolution problem may now be stated as

$$
\min _{\mathbf{s}} .\|\mathbf{r}-\mathbf{B s}\|_{2} \quad \text { subject to } \mathbf{s} \geq 0 \text { and } \mathbf{s} \text { is sparse. }
$$

Note that a solution to this problem provides full information about both the surfaces falling under the laser footprint. Because $\mathbf{s}$ is constrained to be sparse, the location of the non-zero elements provides information about the range to the surfaces. A non-zero element in the $k$ th position indicates a reflecting surface near the range $R=c / k T$. The value of the non-zero elements provide a measure of the strength of the reflection from the surface.

The statement about the sparsity of $\mathbf{s}$ in (23) is usually expressed in terms of the so called 0-norm $\|\mathbf{s}\|_{0}$, which simply counts the number of non-zero elements in a vector. The value of $\|\mathbf{s}\|_{0}$ in (21) is 4 because there are exactly 4 non-zero elements in the vector. A sparse vector will generally have a small value for $\|\mathbf{s}\|_{0}$. Therefore, one way to formulate the sparse optimization problems is by augmenting the penalty function

$$
\min _{\mathbf{s}}\|\mathbf{r}-\mathbf{B s}\|_{2}+\lambda\|\mathbf{s}\|_{0} \quad \text { subject to } \quad \mathbf{s} \geq 0
$$

where $\lambda$ is a user selectable parameter that is used to weight the relative importance of fitting the data (i.e. making $\|\mathbf{r}-\mathbf{B s}\|_{2}$ small) versus finding a sparse solution (i.e. making $\|\mathbf{s}\|_{0}$ small). Note that setting $\lambda=0$ leads to the non-negative LS problem

$$
\min _{\mathbf{s}} .\|\mathbf{r}-\mathbf{B s}\|_{2} \quad \text { subject to } \quad \mathbf{s} \geq 0 .
$$

Typically LS solutions are dense rather than sparse because all the columns of $\mathbf{B}$ are used to approximate $\mathbf{r}$.

At the other extreme is a very large value for $\lambda$. In this case, the penalty function places so much weight on encouraging the elements of $\mathbf{s}$ to be zero that they all become zero. As $\lambda$ runs the gamut from $\lambda=0$ to large values, the solution $\mathbf{s}$ to (24) starts off dense and becomes more and more sparse until finally the solution is all zeros. Therefore the value of $\lambda$ can be used to encourage $\mathbf{s}$ to be sparse. In the context of deconvolution of ladar data, sparse solutions are physically meaningful. Thus, the optimization in (24) can be used for deconvolution.

It is well known from the theory of compressive sensing and sparse reconstruction ${ }^{10}$ that the 0 -norm regularized problem (24) is NP hard, and it is common to substitute a 1-norm leading to the relaxed problem

$$
\min _{\mathbf{s}} .\|\mathbf{r}-\mathbf{B s}\|_{2}+\lambda\|\mathbf{s}\|_{1} \quad \text { subject to } \quad \mathbf{s} \geq 0
$$

The 1-norm relaxation yields a convex optimization problem ${ }^{13}$ which is easily solved using readily available computer codes. ${ }^{14,15}$ Solutions to the modified problem (26) approximate the solutions of (24). The utility of the relaxation is mathematical tractability. The quality of the results are evaluated on real ladar data.

The sparsity algorithms were evaluated on a small set of ladar pulses. Figures 4 and 6 show deconvolution results for two example ladar shots at a calibrated target panel consisting of two surfaces separated by $25 \mathrm{~cm}$ (Fig. 4) and by $5 \mathrm{~cm}$ (Fig. 6). The data were oversampled by 4, giving the estimated coefficients the physical meaning of range measurements quantized to approximately $(7.5 \mathrm{~cm}) / 4=1.875 \mathrm{~cm}$ resolution. These results show that the solution to unconstrained LS problems tend to be dense (all coefficients are non-zero). Enforcing the non-negativity constraint alone leads to solutions in which many coefficients are zero. The dominant coefficients provide information about the range to the targets (and the distance between surfaces). The non-negatively constrained solution still leaves spurious non-zero elements that may be detected as false reflections. The nonnegative LS results correspond to $\lambda=0$ in the 1-norm regularlized non-negative LS problem (26). To further reduce these spurious coefficients and drive toward more sparse solutions, the $\lambda$ parameter is increased. As $\lambda$ increases from 0 to larger values, the spurious coefficients shrink to zero and solution becomes more and more sparse. Note that in the $25 \mathrm{~cm}$ separation example, raising $\lambda$ does not significantly alter the retrieval of the 25 $\mathrm{cm}$ separation between surfaces. This may be observed in Fig. 5 where the gap between the dominant coefficients is about 14 which corresponds to a physical separation of $14 \times 1.875 \mathrm{~cm}=26.25 \mathrm{~cm}$, a close match to the $25 \mathrm{~cm}$ of actual separation. 


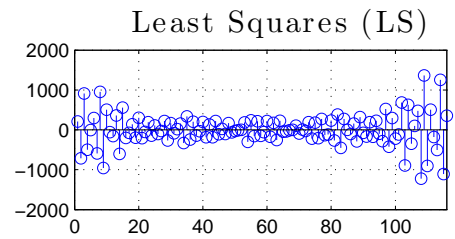

Nonneg. Least Squares (NNLS)

L1-Reg. NNLS, $\lambda=0.4000$
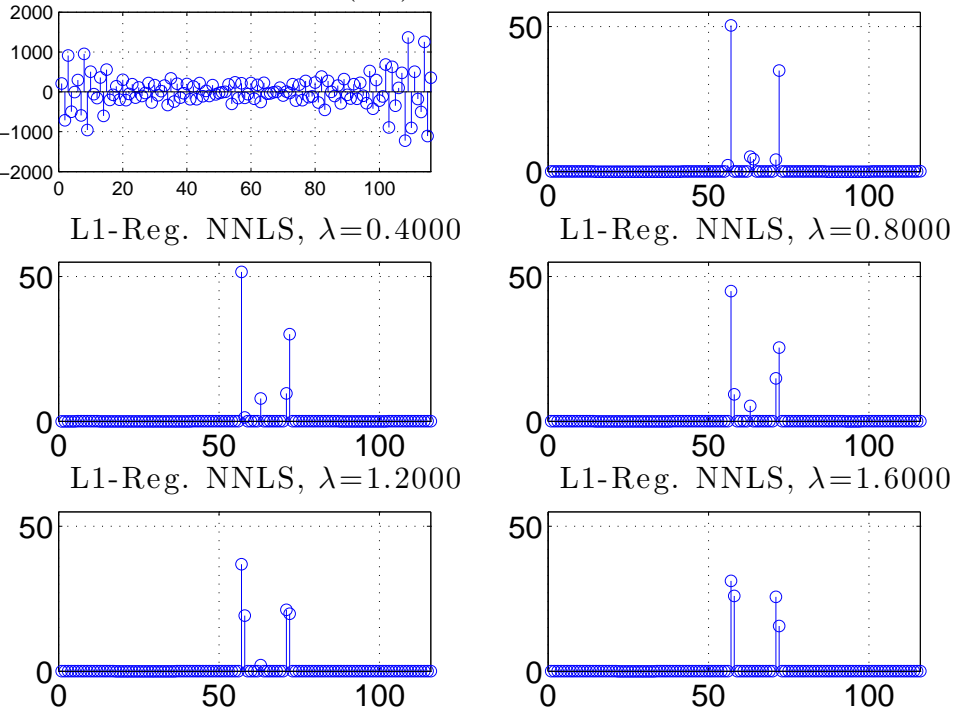

Figure 4. Deconvolution results for surfaces separated by $25 \mathrm{~cm}$ for six LS based algorithms: unconstrained LS (top left), non-negative LS (top right), and for 1-norm regularized non-negative LS using four different values for the regularization.

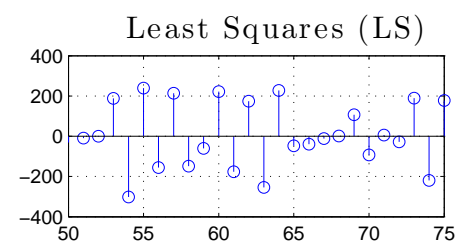

Nonneg. Least Squares (NNLS)

L1-Reg. NNLS, $\lambda=0.4000$

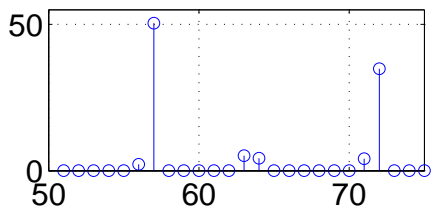

L1-Reg. NNLS, $\lambda=0.8000$
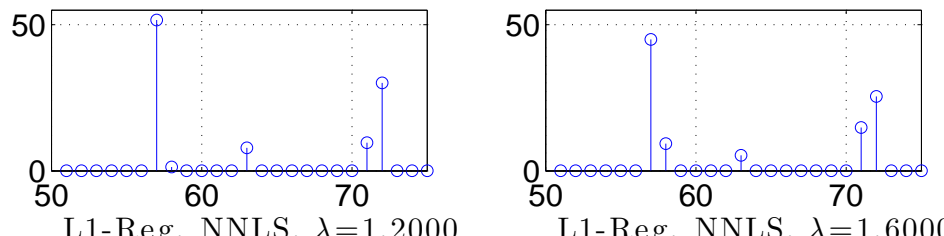

L1-Reg. NNLS, $\lambda=1.2000$
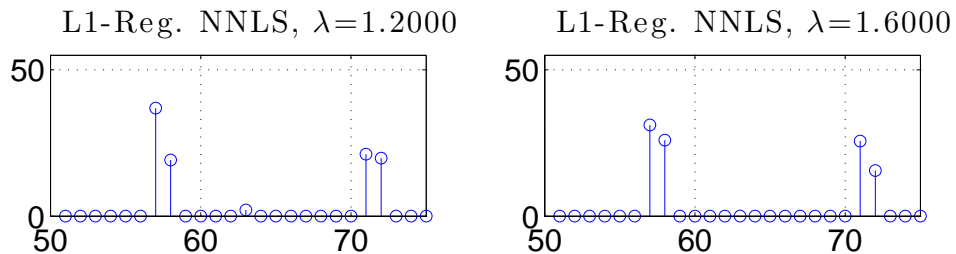

Figure 5. Same as Fig. 4 but zoomed to see detail around the dominant coefficients.

In the $5 \mathrm{~cm}$ separation example, the zoomed detail in Fig. 7 shows the dominant NNLS coefficients are separated by about 6 coefficients which corresponds to $6 \times 1.875 \mathrm{~cm}=11.25 \mathrm{~cm}$ of estimated separation. As $\lambda$ increases to $\lambda=1.6$, the gap decreases to 3 coefficients corresponding to $3 \times 1.875 \mathrm{~cm}=5.625 \mathrm{~cm}$ of estimated separation. In this case, we see that regularization improves the estimation accuracy and gives a good approximation to the actual separation of $5 \mathrm{~cm}$.

\section{EXPERIMENT ON DECONVOLUTION TECHNIQUES}

To measure the ability to resolve two surfaces, a flat target was placed 493 meters from the ELT. The face of the target had a $20 \mathrm{~cm} \times 20 \mathrm{~cm}$ square hole, or slot, cut out from the middle. The area surrounding the slot 


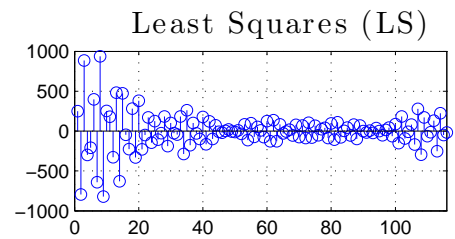

Nonneg. Least Squares (NNLS)

L1-Reg. NNLS, $\lambda=0.4000$

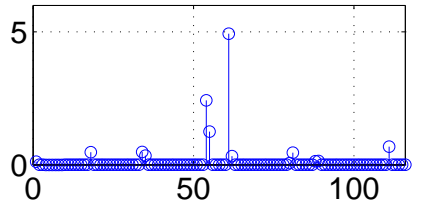

L1-Reg. NNLS, $\lambda=0.8000$
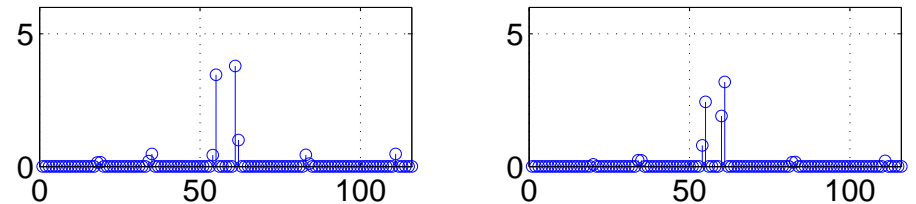

L1-Reg. NNLS, $\lambda=1.2000$

L1-Reg. NNLS, $\lambda=1.6000$
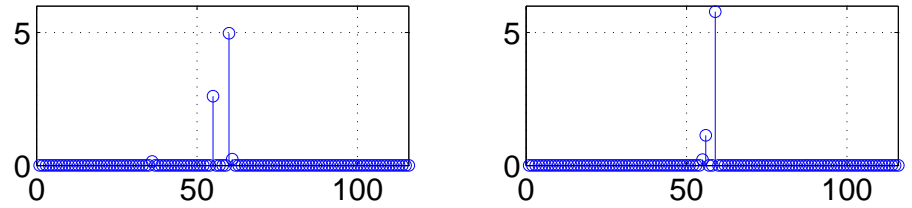

Figure 6. Deconvolution results for surfaces separated by $5 \mathrm{~cm}$ for six LS based algorithms: unconstrained LS (top left), non-negative LS (top right), and for 1-norm regularized non-negative LS using four different values for the regularization.

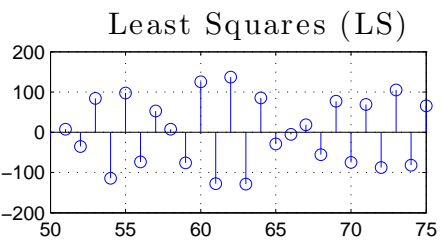

Nonneg. Least Squares (NNLS)

L1-Reg. NNLS, $\lambda=0.4000$
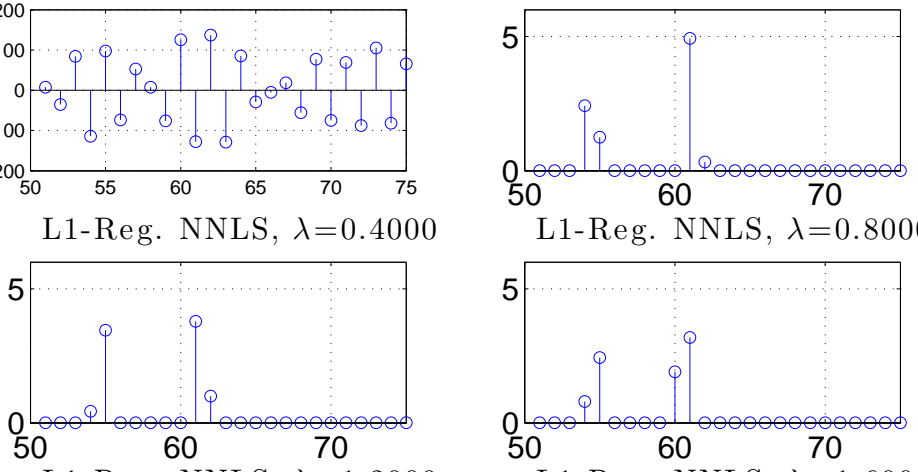

L1-Reg. NNLS, $\lambda=0.8000$
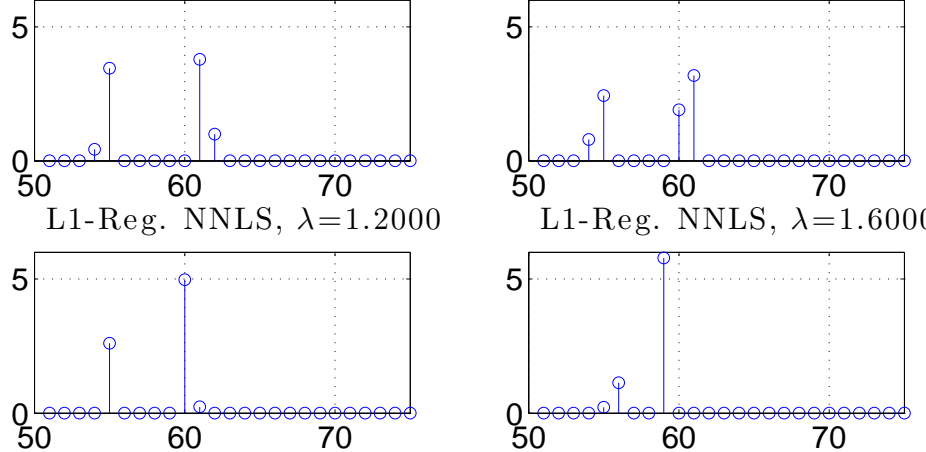

Figure 7. Same as Fig. 6 but zoomed to see detail around the dominant coefficients. 


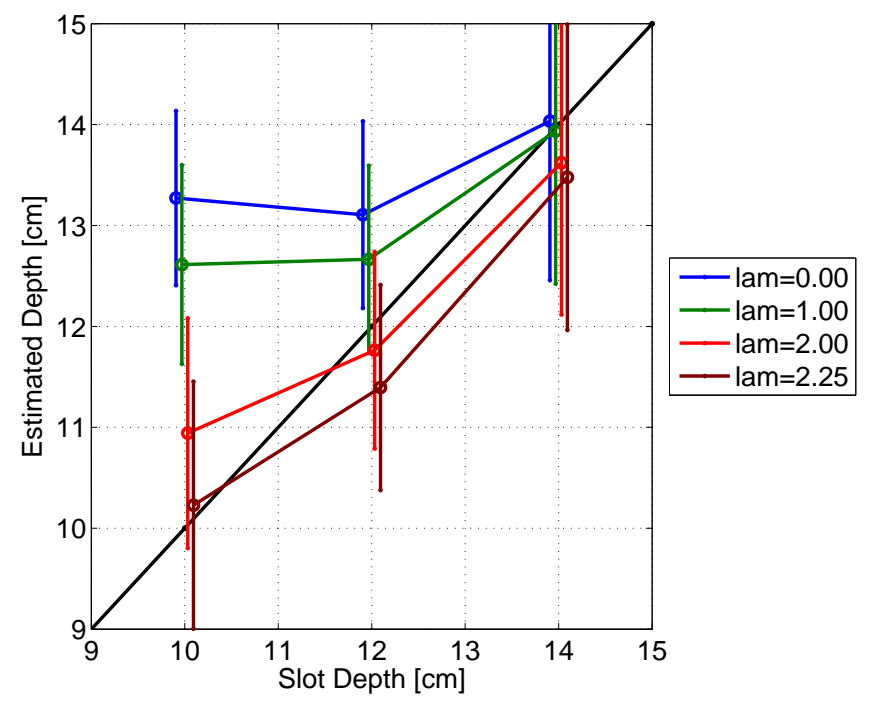

Figure 8. Estimated depth of the slot. The value of $\lambda=0$ is the non-negative LS solution. Circles mark the mean values and error bars mark one standard deviation. (The values are slightly offset to prevent overlapping symbols.)

was large enough to act as a front surface. A second surface was placed directly behind the slot, which was adjustable to different depths, creating two surfaces that have a variable distance between them.

To obtain shots that hit both surfaces, the ELT scanner was set to scan across the slot in the board. From the pointcloud, ten or more shots that hit both surfaces were found and processed using the different deconvolution techniques. Figure 8 shows the results of the experiment as the distance between the two surfaces varied between $14 \mathrm{~cm}$ and $10 \mathrm{~cm}$. These surface separations are at values at the low end of resolution performance obtained previously, ${ }^{8}$ and allow for better understanding of the different sparse deconvolution methods investigated. In the figure, circles mark the mean of the shot depth, and vertical error bars mark one standard deviation in the measurements. The position of the points in the figure were offset slightly to prevent overlap from obscuring the different cases.

The plots show that as the slot depth decreases, the non-negative LS solution diverges from the true value and remains at about $13 \mathrm{~cm}$. In contrast, the sparsity algorithms continue to estimate a decreasing depth. Although some bias is present, the best performance is obtained when $\lambda$ is between 2.0 and 2.25. The variance of the measurements, as marked by error bars, indicates that the uncertainty of the measurements is less than $1.5 \mathrm{~cm}$, and is approximately the same as the depth decreases.

An example of the point cloud extracted from the waveform data taken at a slot depth of $14 \mathrm{~cm}$ is given in Figure 9. Red points represent the location of first returns, and blue points are from second returns. Note that the second returns appear at the edge of the slot. Of interest, but not yet understood, is the slight amount of "splitting" of the single returns in the center of the slot visible in the figure.

An example of a processed return waveform with detections is given in Figure 10, where both a $10 \mathrm{~cm}$ depth and a $14 \mathrm{~cm}$ depth are shown. A constant fraction method was used to detect the returns. ${ }^{16}$ To prevent multiple false detections that are possible if the deconvolved surface response alone is used (see Figures 5 and 7 ), frequency domain regularization is also applied as discussed by Neilsen, ${ }^{17}$ with a bandwidth of about $\frac{1}{4}$ of the upsampled rate.

\section{CONCLUSION}

The LS solution to the deconvolution problem leads to a dense solution that is of little use. This is because of the noise in the system, which leads to the spurious deconvolution signal is matched with no constraint. When the non-negative constraint is applied, which meets physical constraints of the surface response, the solution becomes sparse. However, when additional sparsity constraints are applied with a reasonable level of weighting, 


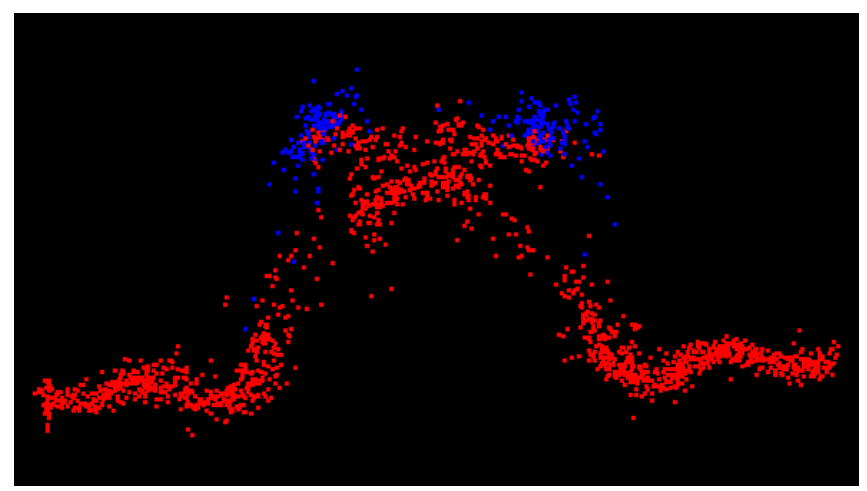

Figure 9. Example point cloud from detections. The view is from the top looking at several scans across the slot. Red points are first returns, blue points are second returns.
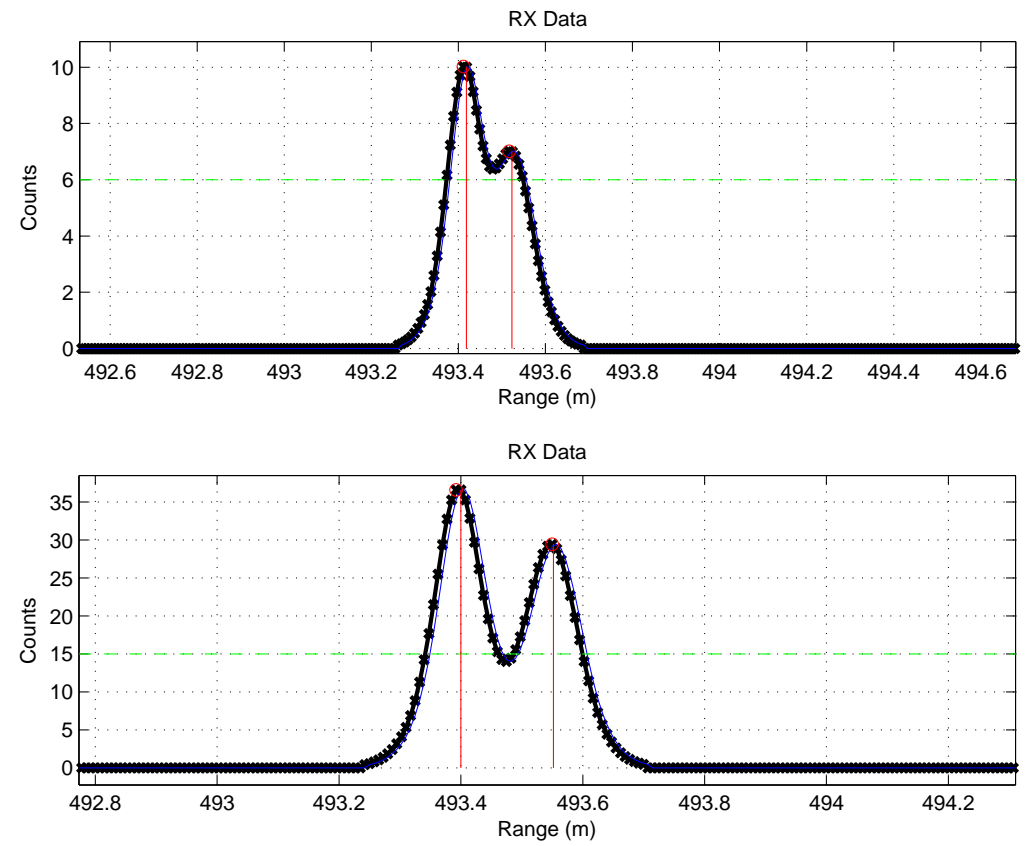

Figure 10. Example pulses with two detections. The detected signal with a separation of $10 \mathrm{~cm}$ (top), and the signal with a separation of $14 \mathrm{~cm}$ (bottom). The red vertical lines mark the detection points. 
the solution moves to a more sparse solution and has the potential to improve the solution. Experiments indicate that the solution at small surface separations is improved slightly.

The resolution of the surface response is affected by the sample rate. The original signal is sampled at a rate that contains only a few samples per pulse, spaced at $7.5 \mathrm{~cm}$ in range. When the signal is upsampled and sparse deconvolution is applied, the resolution improves and the variance of the solution is less than $1 / 3$ of the original sample distance. The effect on upsample rate remains a parameter for study in sparse deconvolution of ladar signals.

\section{REFERENCES}

[1] Mallet, C. and Bretar, F., "Full-waveform topographic lidar: State-of-the-art," ISPRS Journal of Photogrammetry 83 Remote Sensing 64, 1-16 (Jan. 2009).

[2] Neuenschwander, A., Magruder, L., and Gutierrez, R., "Signal processing techniques for feature extraction and classification using small-footprint full-waveform airborne LIDAR," in [Proceedings of IEEE International Geoscience and Remote Sensing Symposium], 3, 676-679, IEEE (July 2008).

[3] Wagner, W., Ullrich, A., Ducic, V., Melzer, T., and Studnicka, N., "Gaussian decomposition and calibration of a novel small-footprint full-waveform digitising airborne laser scanner," ISPRS Journal of Photogrammetry Es Remote Sensing 60, 100-112 (2006).

[4] Jung, J. and Crawford, M. M., "A two-stage approach for decomposition of ICESat waveforms," in [Proceedings of IEEE International Geoscience and Remote Sensing Symposium], 3, 680-683 (July 2008).

[5] Jutzi, B. and Stilla, U., "Range determination with waveform recording laser systems using a Wiener Filter," ISPRS Journal of Photogrammetry $\&$ Remote Sensing 61(2), 95 - 107 (2006).

[6] Jutzi, B., Neulist, J., and Stilla, U., "Sub-pixel edge localization based on laser waveform analysis," in [International Archives of Photogrammetry, Remote Sensing and Spatial Information Sciences], 36(Part /W8), 109-114, JSPRS (2005).

[7] Kirchhof, M., Jutzi, B., and Stilla, U., "Iterative processing of laser scanning data by full waveform analysis," ISPRS Journal of Photogrammetry \& Remote Sensing 63, 99-114 (2008).

[8] Neilsen, K. D., Budge, S. E., and Pack, R. T., "Signal processing on waveform data from the eyesafe ladar testbed (ELT)," in [Laser Radar Technology and Applications XV], Turner, M. D. and Kamerman, G. W., eds., 7684, 76840R, SPIE, Orlando, Florida, USA (Apr. 2010).

[9] Marquardt, D. W., "An algorithm for least-squares estimation of nonlinear parameters," Journal of the Society for Industrial and Applied Mathematics 11(2), 431-441 (1963).

[10] Elad, M., [Sparse and Redundant Representations: From Theory to Applications in Signal and Image Processing], Springer (2010).

[11] Pack, R. T., Swasey, J. A., Fullmer, R. R., Budge, S. E., Israelsen, P. D., Petersen, B., and Cook, T. D., "Eyesafe LADAR test-bed with coaxial color imager," in [Laser Radar Technology and Applications XIV], Turner, M. D. and Kamerman, G. W., eds., 7323, 732303, SPIE, Orlando, FL, USA (Apr. 2009).

[12] Pack, R. T., Israelsen, P., and Sealy, K., "A co-boresighted synchronized ladar/EO imager for creating 3D images of dynamic scenes," in [Laser Radar Technology and Applications X], Kamerman, G. W., ed., 5791, 42-50, SPIE (May 2005).

[13] Boyd, S. and Vandenberghe, L., [Convex Optimization], Cambridge (2004).

[14] Grant, M. and Boyd, S., "CVX: Matlab software for disciplined convex programming, version 2.1." http: //cvxr.com/cvx (Mar. 2014).

[15] Grant, M. and Boyd, S., "Graph implementations for nonsmooth convex programs," in [Recent Advances in Learning and Control], Blondel, V., Boyd, S., and Kimura, H., eds., Lecture Notes in Control and Information Sciences, 95-110, Springer-Verlag Limited (2008). http://stanford.edu/ boyd/graph_dcp. html.

[16] Kilpelä, A., Pulsed Time-of-Flight Laser Range Finder Techniques for Fast, High Precision Measurement Applications, $\mathrm{PhD}$ thesis, University of Oulu (2004).

[17] Neilsen, K. D. and Budge, S. E., "Enhanced resolution edge and surface estimation from ladar point clouds containing multiple return data," Opt. Eng. 52, 113103 (Nov. 2013). 